Revista Iberoamericana de las Ciencias Biológicas

y Agropecuarias

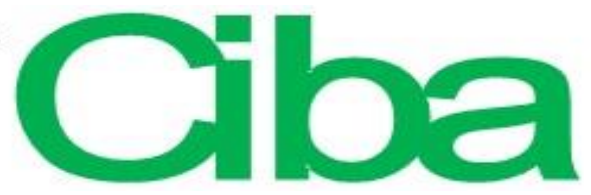

\title{
Comparativa de la eficiencia entre un sistema fotovoltaico con seguimiento solar y un sistema fotovoltaico fijo
}

Comparison between a photovoltaic solar tracker efficiency and a fixed photovoltaic system

Comparação da eficiência entre um sistema fotovoltaico com rastreamento solar e um sistema fotovoltaico fixo

Jorge Arturo Pelayo López

Centro Universitario de la Costa Sur de la Universidad de Guadalajara, México jorgep@cucsur.udg.mx https://orcid.org/0000-0003-3470-9176

\begin{abstract}
Alfredo Luna Soto
Centro Universitario de la Costa Sur de la Universidad de Guadalajara, México alfred@cucsur.udg.mx https://orcid.org/0000-0002-0075-2152

Francisco Bernabe Ramos

Centro Universitario de la Costa Sur de la Universidad de Guadalajara, México fbernabe@cucsur.udg.mx https://orcid.org/0000-0002-7365-5257

Benjamín Guzmán Flores Centro Universitario de la Costa Sur de la Universidad de Guadalajara, México benjamin@cucsur.udg.mx https://orcid.org/0000-0002-0430-1359
\end{abstract}




\section{Revista Iberoamericana de las Ciencias Biológicas y Agropecuarias}

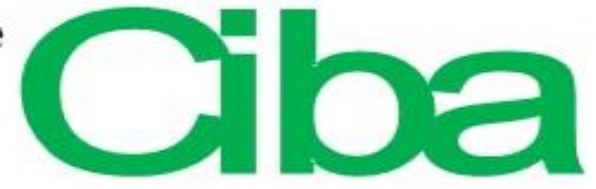

\section{Resumen}

Para generar de forma eficiente energía eléctrica utilizando paneles fotovoltaicos es indispensable que estos sean instalados de forma correcta. Para ello, se pueden implementar sistemas de control de posicionamiento (seguidor solar) mediante un algoritmo de búsqueda del punto máximo de energía, lo cual sirve para mejorar la eficiencia del sistema. Por tal motivo, en el presente trabajo se analiza el diseño y la construcción de un sistema fotovoltaico con seguimiento solar de dos ejes. El objetivo es determinar la eficiencia de este sistema frente a uno estático. El seguidor solar construido cuenta con celdas independientes que actúan como sensores y alimentan a los motores encargados de girar el panel fotovoltaico tanto en el eje vertical como en el horizontal. Para la adquisición de la energía generada por los dos sistemas de paneles solares se utilizó la tarjeta Arduino Nano 3.0 y diversos módulos. Los resultados de las pruebas realizadas se examinaron mediante el programa computacional SigmaPlot y la comparativa de grupos (ANOVA) de una vía. Asimismo, se realizó una prueba de rangos múltiples, que emplea el método de comparación múltiple de medias de Tukey. Luego se confrontaron los datos recabados durante un periodo de 29 días. Los resultados demostraron que en ese lapso la eficiencia promedio alcanzada por el sistema con seguimiento solar fue de $33 \%$, mientras que con el sistema fijo fue de $26.28 \%$. Además, se observó que, durante las primeras horas de cada día, el sistema fotovoltaico fijo logró generar más energía eléctrica que el sistema fotovoltaico con seguimiento solar.

Palabras clave: eficiencia, potencia, seguidor solar, sistema fijo, sistema fotovoltaico.

\section{Abstract}

The optimal installation of photovoltaic panels plays an important role for the efficient generation of electrical energy. To find the maximum energy point of a photovoltaic panel, the positioning control system (solar tracker) can be implemented by means of a technique or a search algorithm to improve the generation system efficiency. In the present work the design and construction of two shafted photovoltaic solar tracker system was addressed. The aim is to determine the efficiency of this system and compare with a fixed or static photovoltaic system. The solar tracker has independent cells that act as sensors and feed the DC motors responsible of turning the photovoltaic panel in both the vertical and the horizontal axis. For the acquisition of the power generated by 


\section{Revista Iberoamericana de las Ciencias Biológicas y Agropecuarias}

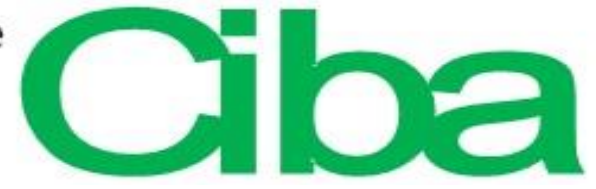

each solar panel, an Arduino Nano 3.0 card and different modules were used, as well as the environment itself Arduino programming was also utilized. The results were examined through the SigmaPlot computer program. These results were analyzed by comparing groups (ANOVA) in one way, followed by a multiple range test, which uses Tukey's multiple measures comparison method. Field tests were conducted for a period of 29 days, and the results of both systems were compared. The results showed that, the average efficiency reached by the system with solar tracking during the experimental period, was 33\%, while the fixed system achieved an average efficiency of $26.28 \%$, therefore it is deduced that the percentage of the efficiency of the solar tracker system for this period was greater than the fixed system by $7 \%$, approximately. Finally, it was observed that, during the first hours of each day, the fixed photovoltaic system managed to generate more electricity than the photovoltaic system with solar tracking.

Keywords: efficiency, power, solar tracker, fixed system, photovoltaic system.

\section{Resumo}

Para gerar energia elétrica eficientemente usando painéis fotovoltaicos, é essencial que eles sejam instalados corretamente. Para isso, sistemas de controle de posicionamento (rastreador solar) podem ser implementados por meio de um algoritmo de busca para o ponto máximo de energia, que serve para melhorar a eficiência do sistema. Por este motivo, no presente trabalho é analisado o projeto e a construção de um sistema fotovoltaico com rastreamento solar de dois eixos. O objetivo é determinar a eficiência deste sistema contra um sistema estático. O rastreador solar integrado possui células independentes que atuam como sensores e alimentam os motores responsáveis por girar o painel fotovoltaico no eixo vertical e horizontal. Para a aquisição da energia gerada pelos dois sistemas de painéis solares, utilizou-se a placa Arduino Nano 3.0 e vários módulos. Os resultados dos testes realizados foram examinados através do programa de computador SigmaPlot e da comparação de grupo unidirecional (Anova). Da mesma forma, foi realizado um teste de múltiplas faixas, que utiliza o método de comparação de médias múltiplas de Tukey. Em seguida, os dados coletados durante um período de 29 dias foram comparados. Os resultados mostraram que, nesse período, a eficiência média alcançada pelo sistema de rastreamento solar foi de $33 \%$, enquanto no sistema fixo foi de 26,28\%. Além disso, observou-se 


\section{Revista Iberoamericana de las Ciencias Biológicas y Agropecuarias}

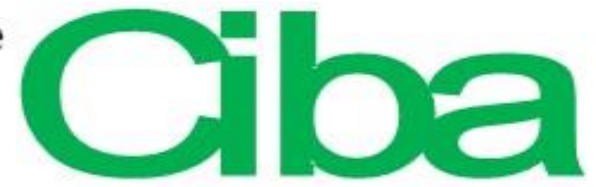

que durante as primeiras horas de cada dia o sistema fotovoltaico fixo conseguiu gerar mais energia elétrica do que o sistema fotovoltaico com rastreamento solar.

Palavras-chave: eficiência, energia, rastreador solar, sistema fixo, sistema fotovoltaico.

Fecha recepción: Julio 2017

Fecha aceptación: Enero 2018

\section{Introducción}

La generación de energía es uno de los temas ambientales más importantes de la actualidad. Esto se debe, principalmente, al uso indiscriminado de combustibles fósiles para obtener electricidad, lo cual representa más de $80 \%$ de la energía producida en el mundo (Dick Hedberg, 2010; Noa-Diéguez, Álvarez-Sánchez y Pérez-Rodríguez, 2015). De acuerdo con las estadísticas de la Agencia Internacional de Energía (IEA, por sus siglas en inglés), en el planeta se producen 21,431 TWh, y en México 259 TWh. Esto significa que $75 \%$ de la energía originada a nivel mundial proviene de materias como el carbón, el petróleo y el gas natural. En México esta proporción es más elevada, aproximadamente 77 \% (International Energy Agency [IEA], 2010).

Ahora bien, la masiva explotación de fuentes no renovables ha provocado consecuencias catastróficas, como el efecto invernadero, el calentamiento global y el deterioro de ecosistemas terrestres y marinos. Ante esta situación, cada vez es más acentuado el interés por emplear fuentes limpias, de las cuales la radiación solar es una de las que tiene mayor potencial - especialmente en las regiones intertropicales - porque puede producir el doble de la energía de los combustibles fósiles. Sin embargo, el problema se halla en que los generadores de la actualidad solo tienen una eficiencia aproximada de $10 \%$ (Mousazadeh et al., 2009). Esta es una de las razones por las que se invierte tanto tiempo y trabajo no solo en la investigación, sino también en el desarrollo de más y mejores tecnologías de energías renovables, como la producida por el viento, el sol, así como la térmica o la generada por biocombustibles (Iglesias y Morales, 2013). 


\section{Revista Iberoamericana de las Ciencias Biológicas y Agropecuarias}

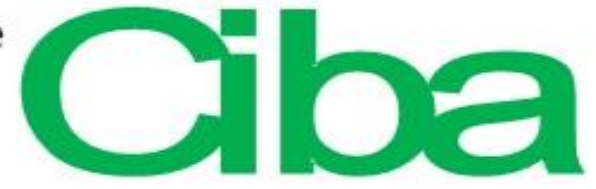

En las últimas décadas, el campo de las fuentes renovables de energía ha cobrado importancia para el desarrollo sustentable. En este contexto, la energía solar se presenta como una alternativa eficiente y económica - en comparación con otras formas tradicionales de energía eléctrica-, de ahí que haya aumentado la necesidad de un mayor aprovechamiento (Machado Toranzo, Lussón Cervantes, Leysdian Oro Carralero, Bonzon Henríquez y Escalona Costa, 2015; Noa-Diéguez et al., 2015).

Según Neha, Gugri, Mishra and Dubey (2013), la cantidad de energía solar recibida anualmente por la tierra representa diez mil veces el consumo del planeta en ese mismo período. Eso significa que el sol —además de ser el actor principal de los procesos biológicos en la tierra— es una poderosa e inagotable fuente de energía, la cual puede ser aprovechada mediante un adecuado sistema de captación y conversión a otro tipo de energía, como la eléctrica, la térmica, entre otras (Arroyo Romero y Cortés Montes de Oca, 2015; Noa-Diéguez et al., 2015).

En este sentido, los paneles fotovoltaicos constituyen uno de los métodos más simples para convertir la energía del sol en electricidad, pues en esta transformación no se crean subproductos peligrosos para el medio ambiente (Domínguez González, 2012). Estos paneles funcionan cuando la luz del sol excita los electrones dentro de las células, lo cual genera energía eléctrica Para ello, no obstante, el ángulo de incidencia de los rayos solares juega un papel determinante, pues una correcta instalación del panel mejora su eficiencia. En efecto, para diferentes valores del ángulo de incidencia en un panel, la salida de este cambiará, de modo que el máximo valor de salida se obtiene cuando los rayos del sol son perpendiculares al panel (Escobar Mejía, Holguín Londoño y Osorio, 2010; Kahn, 2012). Por eso, para encontrar la salida máxima de un panel fotovoltaico se pueden implementar sistemas de control de posicionamiento (seguidor solar) mediante una técnica o un algoritmo de búsqueda del punto máximo de energía para mejorar la eficiencia del sistema (Enríquez, Andújar and Bohórquez, 2010; Escobar Mejía et al., 2010; Grupta, 2011; Panait and Tudorache, 2008).

El seguidor solar es un aparato tecnológico cuya función es aumentar la producción de energía. Para eso, utiliza paneles fotovoltaicos y otros dispositivos de concentración por medio de sistemas mecánicos, eléctricos y electrónicos que siguen la trayectoria del sol. De esa manera captura la máxima radiación solar durante el mayor tiempo posible. Los sistemas fotovoltaicos con seguimiento pueden tener uno o dos ejes. Los primeros solo pueden seguir el azimut solar, pero no 


\section{Revista Iberoamericana de las Ciencias Biológicas y Agropecuarias}

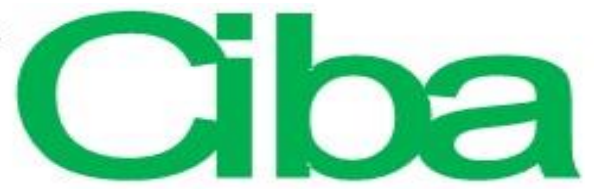

ISSN: $2007-9990$

la altitud solar; mientras que los segundos sí pueden cumplir con ambas funciones, de ahí que sean más eficientes (Ahmet Senpinar, 2012; Koussa, 2011).

Por este motivo, en el presente trabajo se analiza el diseño y la construcción de un sistema fotovoltaico con seguimiento solar de dos ejes. El objetivo es determinar la eficiencia de este cuando se compara con la de un sistema fotovoltaico fijo.

\section{Metodología}

\section{El sistema fotovoltaico con seguimiento solar}

El sistema con seguimiento solar (también llamado seguidor solar) propuesto en este trabajo es de dos ejes, y está conformado por cuatro partes básicas (figura 1):

1. Estructuras de soporte.

2. Sistema de control de movimiento.

3. Sistema de transmisión (etapa de potencia).

4. Sistema de adquisición de datos.

Figura 1. Estructura y sistemas del seguidor solar

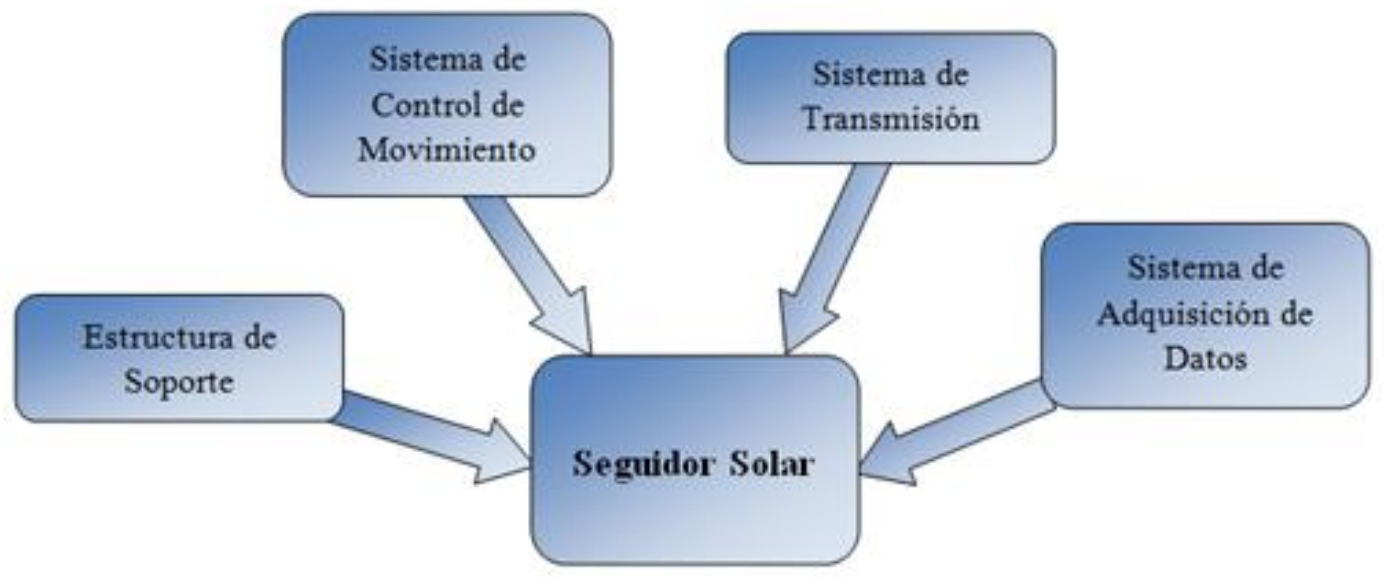

Fuente: Elaboración propia 


\section{Revista Iberoamericana de las Ciencias Biológicas y Agropecuarias}

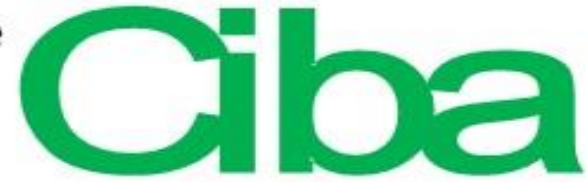

\section{Estructura de soporte}

La estructura metálica que soporta al sistema fotovoltaico con seguimiento solar construido es de material tubular de metal, ángulos de metal y perfil tubular rectangular. Esto brinda firmeza al seguidor solar, soporta tanto al sistema de control de movimiento como al de transmisión, y ofrece puntos de unión y agarre de estos. Las figuras 2 (a), 2 (b), 2 (c), 2 (d) y 2 (e) muestran el diseño realizado mediante el software SolidWorks.

Figura 2. Estructura de soporte para el sistema fotovoltaico con seguimiento solar

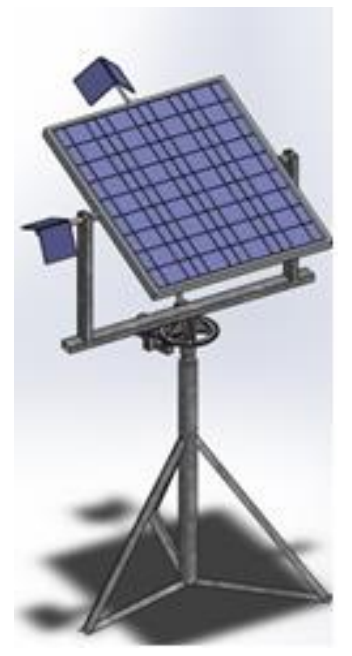

a

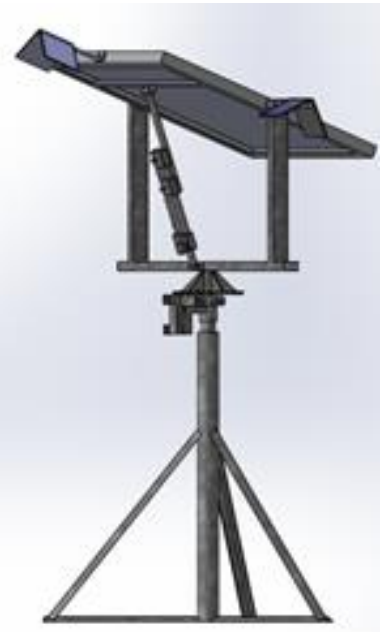

b

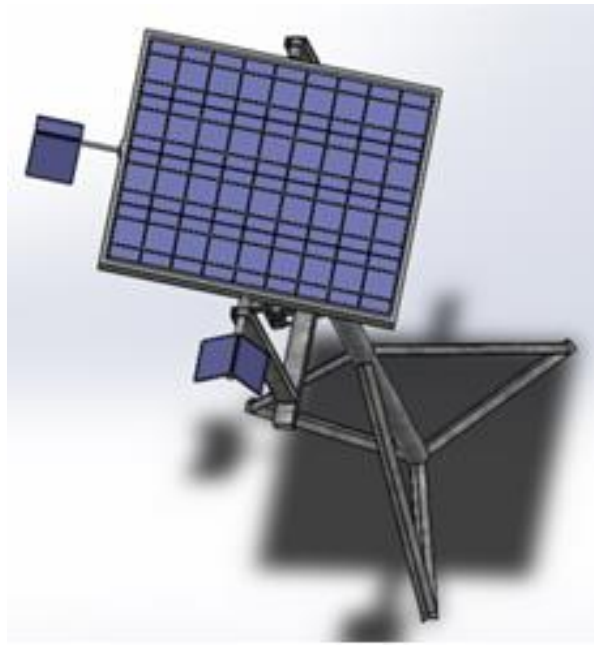

c

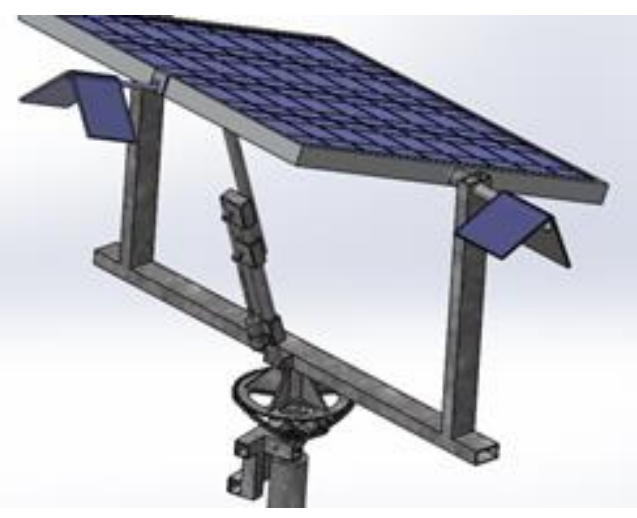

d

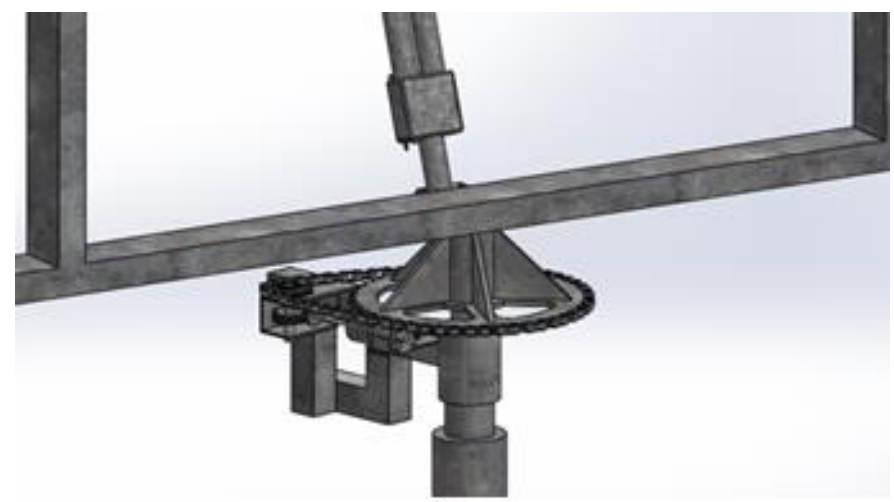

e

Fuente: Elaboración propia 


\section{Revista Iberoamericana de las Ciencias Biológicas y Agropecuarias}

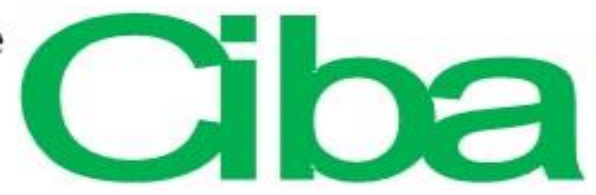

\section{Sistema de control de movimiento}

Este sistema es el encargado de obtener el ángulo acimutal correspondiente al movimiento del eje vertical y el ángulo de declinación o de elevación propio del movimiento del eje horizontal del panel fotovoltaico (figura 3).

Figura 3. Ángulo acimutal y de declinación del seguidor solar

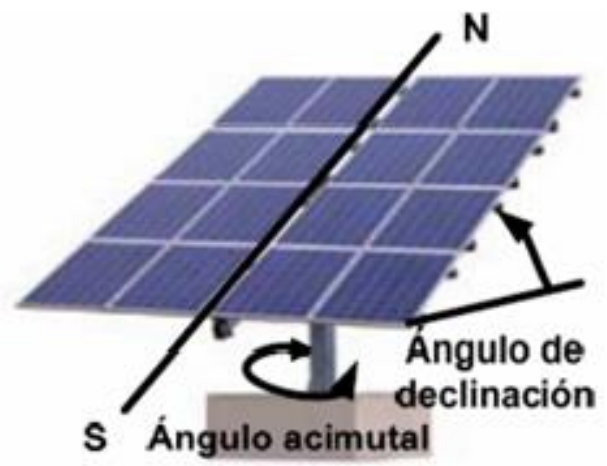

Fuente: Núñez Flores (2012)

A continuación, se explica el sistema que controla los movimientos del panel fotovoltaico. El sistema utiliza un par de celdas solares independientes con especificaciones técnicas de $6 \mathrm{Vcc}$, $110 \mathrm{~mA}$ y $0.6 \mathrm{~W}$ cada una. Estas celdas solares actúan como sensores para proporcionar el movimiento vertical (acimutal); están localizadas una a noventa grados de la otra y se encuentran colocadas en la parte superior del panel solar (figura 4). Dependiendo de cuál de las dos celdas solares recibe más luz, entonces será el movimiento acimutal que va a tener el panel fotovoltaico. Otro par de celdas solares independientes son utilizadas como sensores para proporcionar el movimiento horizontal; estas celdas tienen el mismo funcionamiento que los sensores que dan el movimiento acimutal. 


\section{Revista Iberoamericana de las Ciencias Biológicas y Agropecuarias}

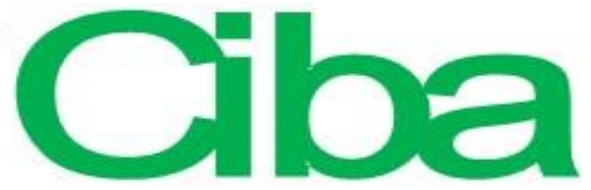

Figura 4. Sensores para el movimiento vertical y horizontal del seguidor solar

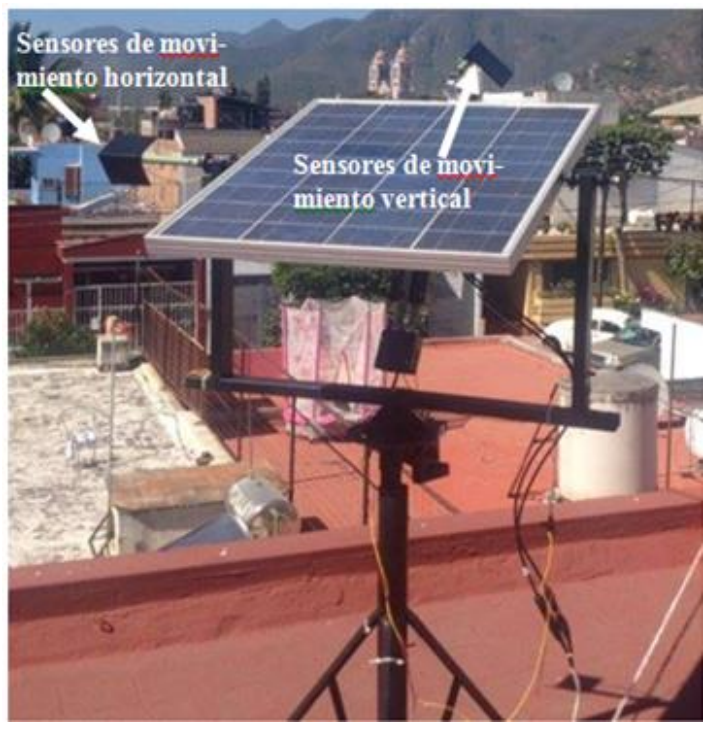

Fuente: Elaboración propia

En la figura 5 se ilustra el diagrama de flujo para el control de movimiento del sistema fotovoltaico construido.

Figura 5. Diagrama de flujo para el control de movimiento del sistema con seguimiento solar

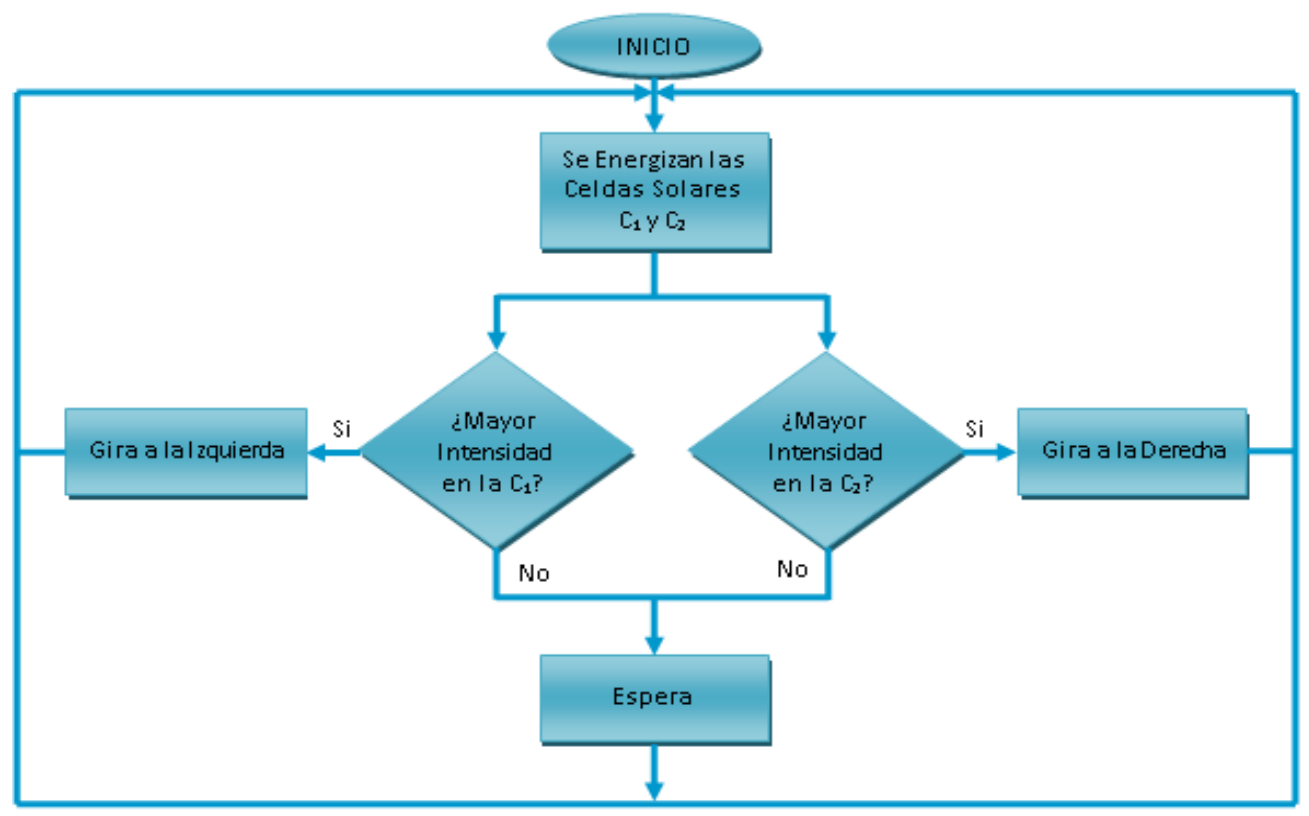

Fuente: Elaboración propia 


\section{Revista Iberoamericana de las Ciencias Biológicas y Agropecuarias}

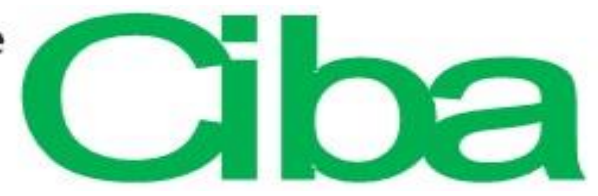

\section{Sistema de transmisión (etapa de potencia)}

Este sistema está constituido por un conjunto de estrellas y cadenas — como se mostró en las figuras 2 (d) y 2 (e) — que se mueven por motores de corriente directa. El seguidor solar utiliza dos motores para su funcionamiento: uno para el movimiento vertical y otro para el movimiento horizontal. El sentido del movimiento de los motores es definido por la diferencia de potencia que emiten las celdas solares. La conexión de las celdas solares a cada uno de los motores que proporcionan los movimientos vertical y horizontal se muestra en la figura 6, y se explica de la siguiente manera: la celda solar 1 se encuentra conectada al motor con polarización directa (positivo de la celda solar con el positivo del motor, y negativo de la celda solar con el negativo del motor); esto ocasiona que cuando el sol irradia a esta celda solar, ella hace girar el motor en el sentido de las manecillas del reloj. La celda solar 2 está conectada en polarización inversa (positivo de la celda solar con el negativo del motor, y negativo de la celda solar con el positivo del motor); cuando el sol irradia a esta segunda celda solar, ella hace girar el motor en el sentido contrario a las manecillas del reloj. Ahora bien, cuando el sol irradia con la misma intensidad a las dos celdas solares, el motor permanece sin movimiento.

Figura 6. Sistema de transmisión para el movimiento vertical y horizontal del panel fotovoltaico

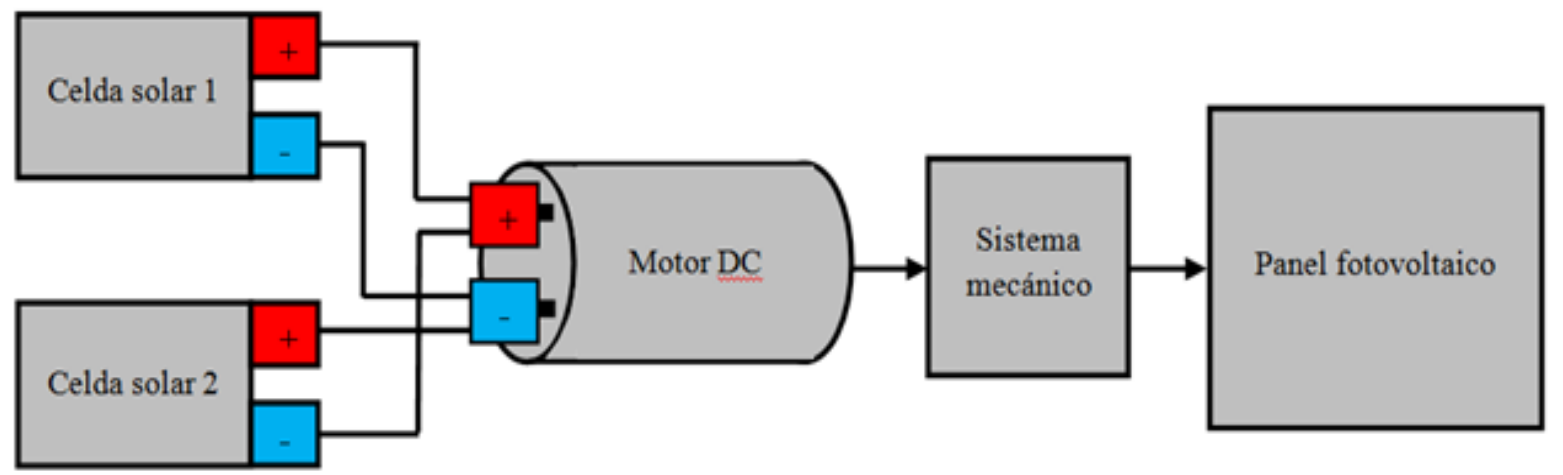

Fuente: Elaboración propia 


\section{Revista Iberoamericana de las Ciencias Biológicas y Agropecuarias}

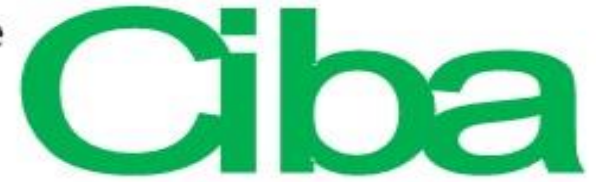

ISSN: 2007 - 9990

\section{Sistema de adquisición de datos}

Para la adquisición tanto del amperaje como del voltaje que emiten cada uno de los dos paneles fotovoltaicos (con seguimiento solar y fijo) se utilizó una tarjeta Arduino Nano 3.0, un sensor de tiempo real DS3231, dos sensores de corriente ACS712 y varios conectores. Estos componentes electrónicos se encuentran interconectados a través de una tarjeta de circuito impreso (del inglés printed circuit board, PCB), a la que se denominó tarjeta de adquisición de datos. En la figura 7 se muestra el diagrama de conexiones de esta tarjeta, la cual se utilizó durante las pruebas de campo para obtener la corriente, el voltaje y, por ende, la potencia generada por ambos sistemas.

Figura 7. Diagrama de conexiones de esta tarjea de adquisición de datos

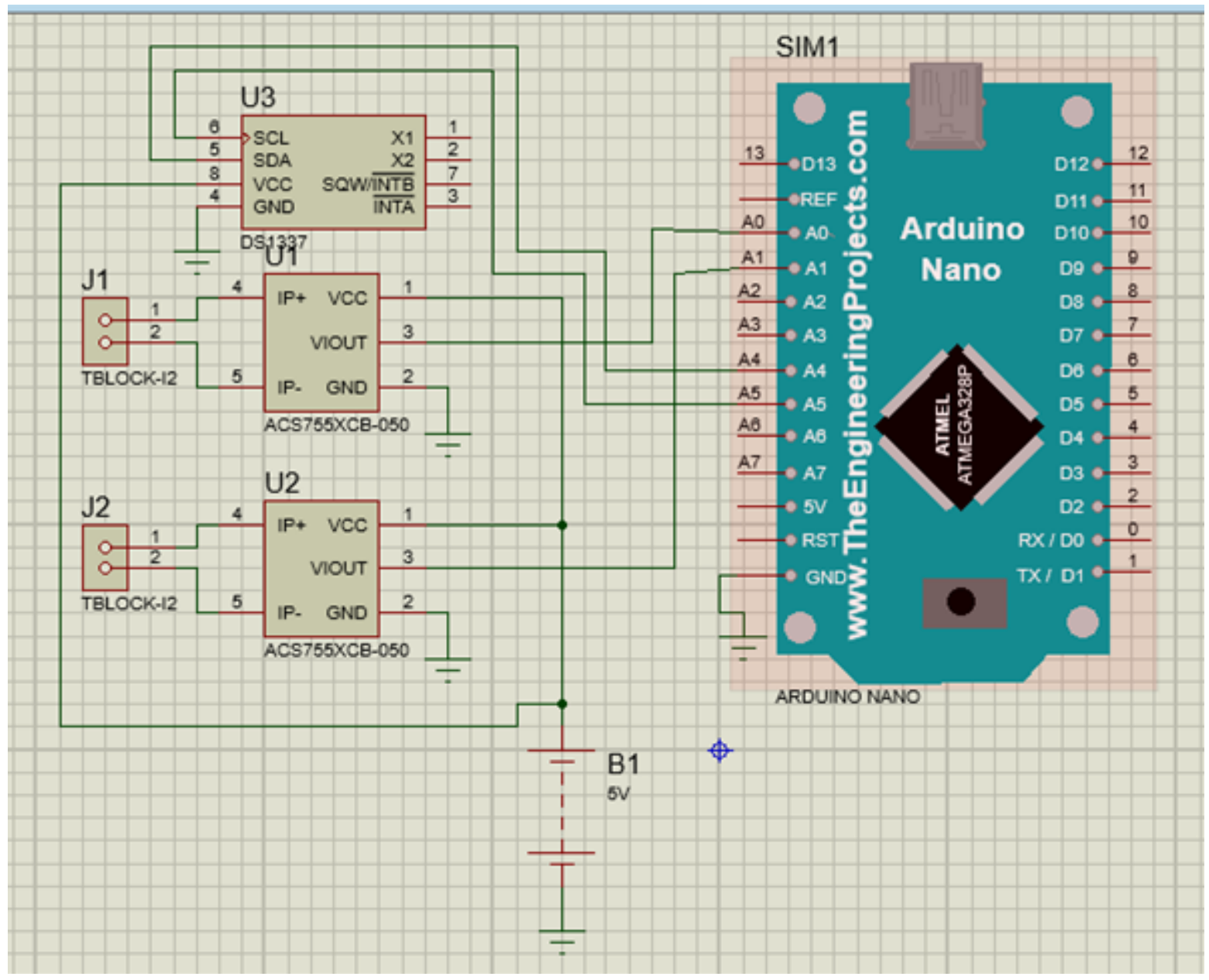

Fuente: Elaboración propia 


\section{Revista Iberoamericana de las Ciencias Biológicas y Agropecuarias}

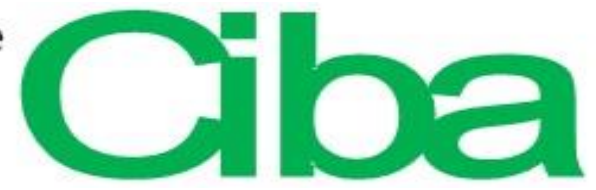

\section{El sistema fotovoltaico fijo}

El sistema fotovoltaico fijo utilizado en este trabajo es un panel fotovoltaico de $65 \mathrm{~W}$ de potencia, posicionado con un ángulo de elevación fijo igual a $19.771^{\circ}$. En la figura 8 se observa este sistema, el cual fue instalado en la ciudad de Autlán de Navarro, Jalisco, para realizar las pruebas de campo de esta investigación. Cabe señalar que el ángulo de elevación de este panel se obtuvo con la ayuda de las aplicaciones SunEarthTools.com y Solar Topo, ambas disponibles en la Red sin costo alguno.

Figura 8. Sistema fotovoltaico fijo

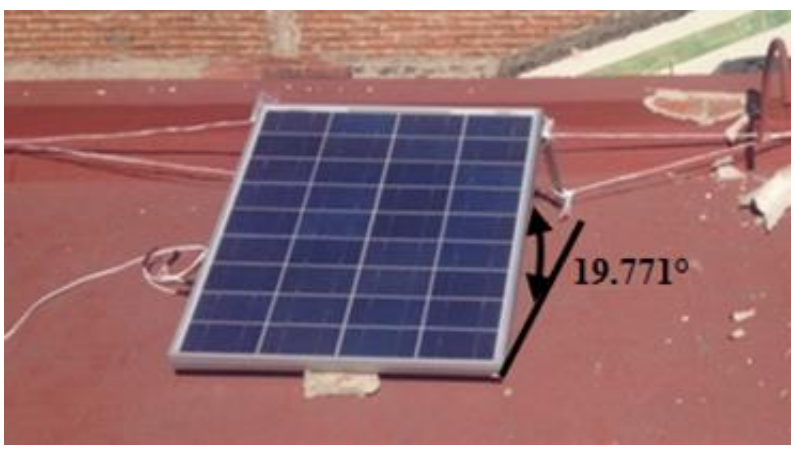

Fuente: Elaboración propia

\section{Desarrollo experimental}

El trabajo de campo de esta investigación se llevó a cabo con la experimentación de los dos sistemas fotovoltaicos tratados en este trabajo (ambos de $65 \mathrm{~W}$ ) durante un periodo de 29 días (del 14 de febrero al 15 de marzo de 2017). Para realizar las pruebas de campo los dos sistemas se instalaron en el domicilio de Adán Uribe 111B, situado en la ciudad de Autlán de Navarro, Jalisco, con ubicación geográfica de $19.771^{\circ}$ de latitud. Para medir el voltaje y la corriente generados se implementó un sistema de adquisición de datos basado en la plataforma Arduino, el cual permitió percibir sus mediciones y calcular simultáneamente la potencia mediante la fórmula 1.

$$
\mathrm{P}=\mathrm{VI}
$$

Donde

$$
\mathrm{P}=\text { Potencia eléctrica }(\mathrm{W})
$$




\section{Revista Iberoamericana de las Ciencias Biológicas y Agropecuarias}

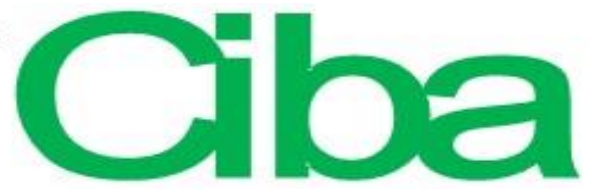

$$
\begin{gathered}
\text { V = Voltaje }(\mathrm{V}) \\
\mathrm{I}=\text { Corriente eléctrica }(\mathrm{A})
\end{gathered}
$$

Para determinar la eficiencia energética se empleó la fórmula 2, la cual está incluida en la norma internacional ISO 50001 sobre sistemas de gestión de la energía, publicada en septiembre de 2011 por la Organización Internacional para la Estandarización (International Organization for Standardization [ISO], 2011).

$$
\text { Eficiencia energética }=\frac{\text { Resultado }}{\text { Entrada total de energía }}
$$

Donde

$$
\text { Resultado = Potencia generada por el panel fotovoltaico }(\mathrm{W})
$$

Entrada total de energía = Potencia máxima del panel fotovoltaico $(\mathrm{W})$

La información obtenida mediante la tarjeta de adquisición de datos (voltaje y corriente) se exportó a una hoja de cálculo de Excel, en donde se realizaron las gráficas del comportamiento de la eficiencia por ambos sistemas fotovoltaicos. La eficiencia está dada en porcentajes, para lo cual se utilizó la fórmula 3. La experimentación se realizó entre las 09:00 horas y las 19:00 horas, ya que antes y después de ese horario se adquirían valores mínimos. El intervalo entre cada una de las mediciones fue de 15 minutos.

$$
\text { Eficiencia energética }=\left(\frac{\text { Resultado }}{\text { Entrada total de energía }}\right) \times 100 \%
$$

Finalmente, para analizar los resultados se utilizó el programa computacional SigmaPlot. Los estadísticos descriptivos reportaron la eficiencia de cada uno de los sistemas fotovoltaicos evaluados por hora y posteriormente por día. Los resultados de cada set experimental fueron examinados para e ncontrar diferencias estadísticas significativas entre los métodos evaluados en cada tratamiento mediante un análisis de comparativa de grupos (Anova) de una vía, seguido de una prueba de rangos múltiples, que emplea el método de comparación múltiple de medias de Tukey. 


\section{Revista Iberoamericana de las Ciencias Biológicas y Agropecuarias}

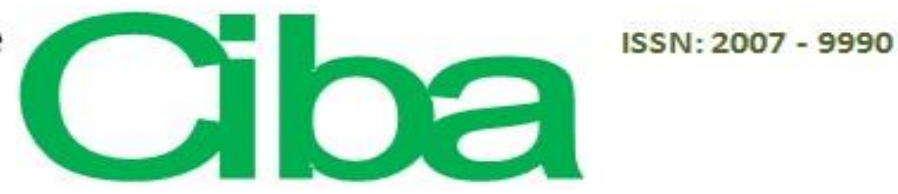

\section{Resultados y discusión}

La información expuesta en este trabajo corresponde a la eficiencia de ambos sistemas de paneles fotovoltaicos en los días más inestables de los meses antes mencionados, es decir, un día parcialmente nublado (19 de febrero de 2017) y un día soleado (14 de marzo del mismo año). En esta sección se presenta la información recabada durante la prueba preliminar y la correspondiente a un día parcialmente nublado y un día soleado, así como la obtenida durante los 29 días del experimento (del 14 de febrero al 15 de marzo de 2017).

\section{Prueba preliminar}

Las diferencias en la eficiencia lograda por los dos sistemas durante la prueba preliminar realizada el 15 de febrero de 2017 se muestran en la figura 9. En esta se puede apreciar que para el rango de tiempo observado el sistema fotovoltaico con seguimiento solar generó mayor potencia eléctrica que el sistema fotovoltaico fijo. De hecho, a las 13:30 horas de ese día el sistema con seguimiento solar alcanzó su máxima eficiencia (65.18 \%), mientras que a las 12:00 horas obtuvo la mayor eficiencia en comparación con el sistema fijo (17.11\%).

Por otro lado, de 10:30 horas a 10:45 horas, y de 13:45 horas a 14:15 horas se registró la misma eficiencia para ambos sistemas debido a que en esos lapsos captaron la misma cantidad de energía producto de una intensa nubosidad.

En cuanto a la eficiencia promedio generada ese día, el sistema con seguimiento solar registró $33.62 \%$, mientras que el sistema fijo consiguió $26.38 \%$ de promedio. Cabe señalar que las caídas en la eficiencia de ambos sistemas (figura 9) corresponden a periodos nublados (ver los registros correspondientes al intervalo de 09:45 horas a 10:15 horas). 
Revista Iberoamericana de las Ciencias Biológicas y Agropecuarias

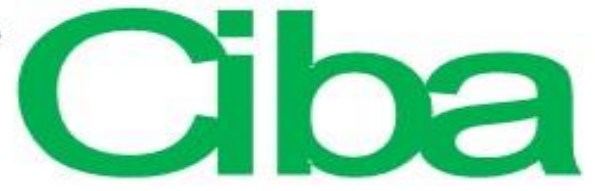

Figura 9. Gráfica del comportamiento de la eficiencia lograda el 15 de febrero de 2017

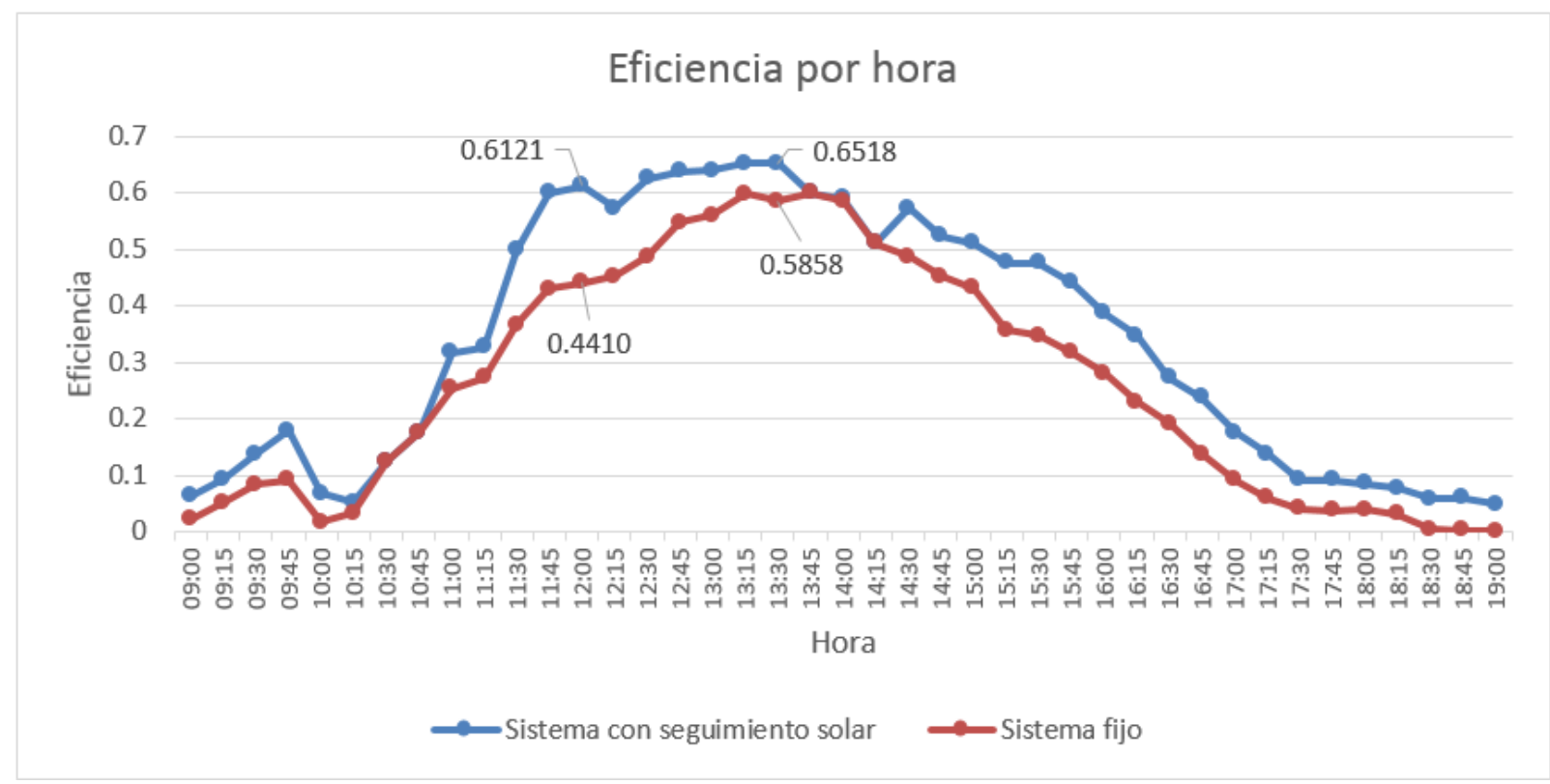

Fuente: Elaboración propia

Por otra parte, el análisis de Tukey para las comparaciones de las medias de ambos sistemas demostró una diferencia mínima significativa entre ellos, como se muestra en las figuras 10 (a) y 10 (b).

Figura 10. Gráficos de la diferencia mínima significativa (Tukey) entre a) el seguidor solar y b) el sistema fijo

a) Seguidor Solar

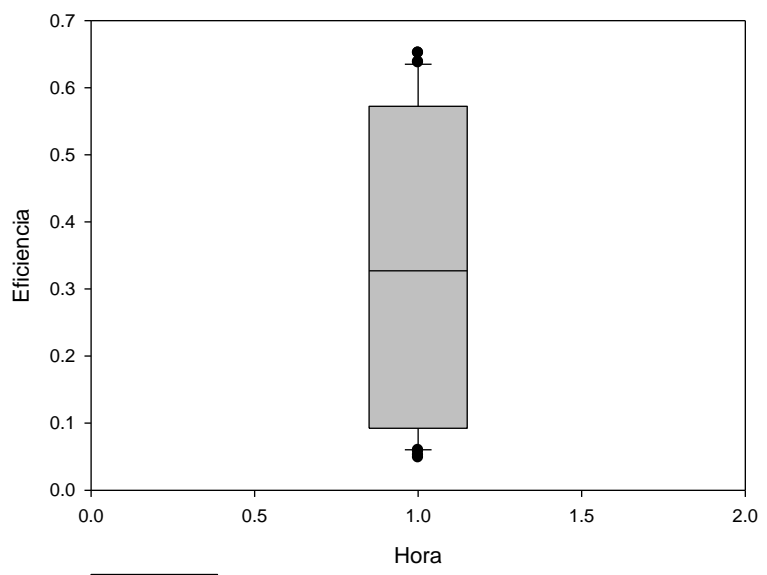

$\square$ Col 1

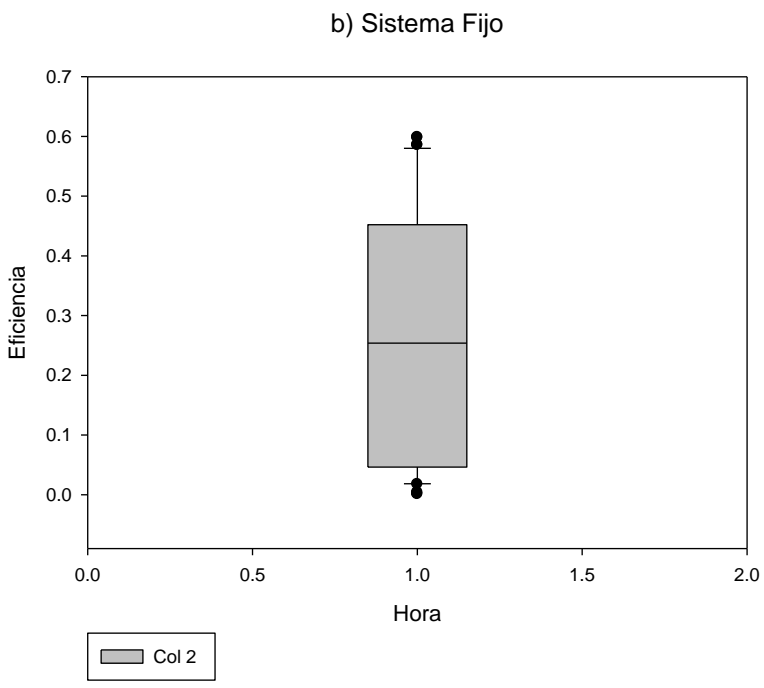

Fuente: Elaboración propia 


\section{Revista Iberoamericana de las Ciencias Biológicas y Agropecuarias}

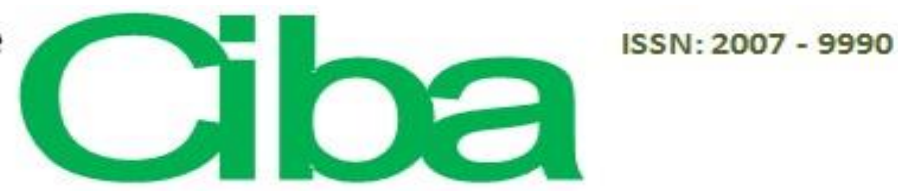

\section{Día parcialmente nublado}

La eficiencia lograda por los dos sistemas fotovoltaicos durante un día parcialmente nublado (19 de febrero de 2017) se muestra en la figura 11. En esta se observa que desde las 09:00 horas hasta las 10:30 horas el sistema fotovoltaico fijo generó mayor potencia eléctrica que el sistema con seguimiento solar. Esto se debió a que todos los días, con la puesta del sol, el panel fotovoltaico con seguimiento solar terminaba orientado hacia el oeste (poniente), de manera que al día siguiente el sol no irradiaba lo suficiente las celdas utilizadas como sensores. Esto ocasionaba que no se transmitiera al motor la energía necesaria para hacer girar el panel fotovoltaico hacia el oriente (donde el sol inicia su recorrido). También se observó que para el resto de este día (excepto algunos periodos donde se presentaron intensas nubosidades) el sistema fotovoltaico con seguimiento solar generó mayor potencia eléctrica que el sistema fotovoltaico fijo. De hecho, a las 14:45 horas de ese día el sistema con seguimiento solar alcanzó su máxima eficiencia (77.44 \%), mientras que a las 15:15 horas registró la mayor eficiencia en comparación con el sistema fijo $(17.36 \%)$.

En cuanto a la eficiencia promedio generada ese día, el sistema con seguimiento solar registró $30.72 \%$, mientras que el sistema fijo consiguió $24.42 \%$ de promedio. Este dato demuestra que en días con diversas nubosidades el sistema con seguimiento solar puede generar mayor energía que el sistema fijo. 


\section{Revista Iberoamericana de las Ciencias Biológicas y Agropecuarias}

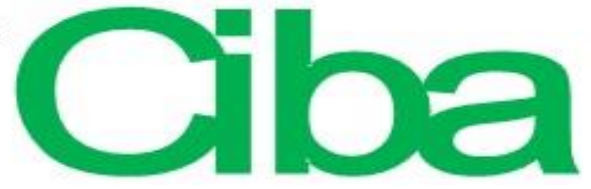

Figura 11. Gráfica del comportamiento de la eficiencia obtenida el día 19 de febrero de 2017

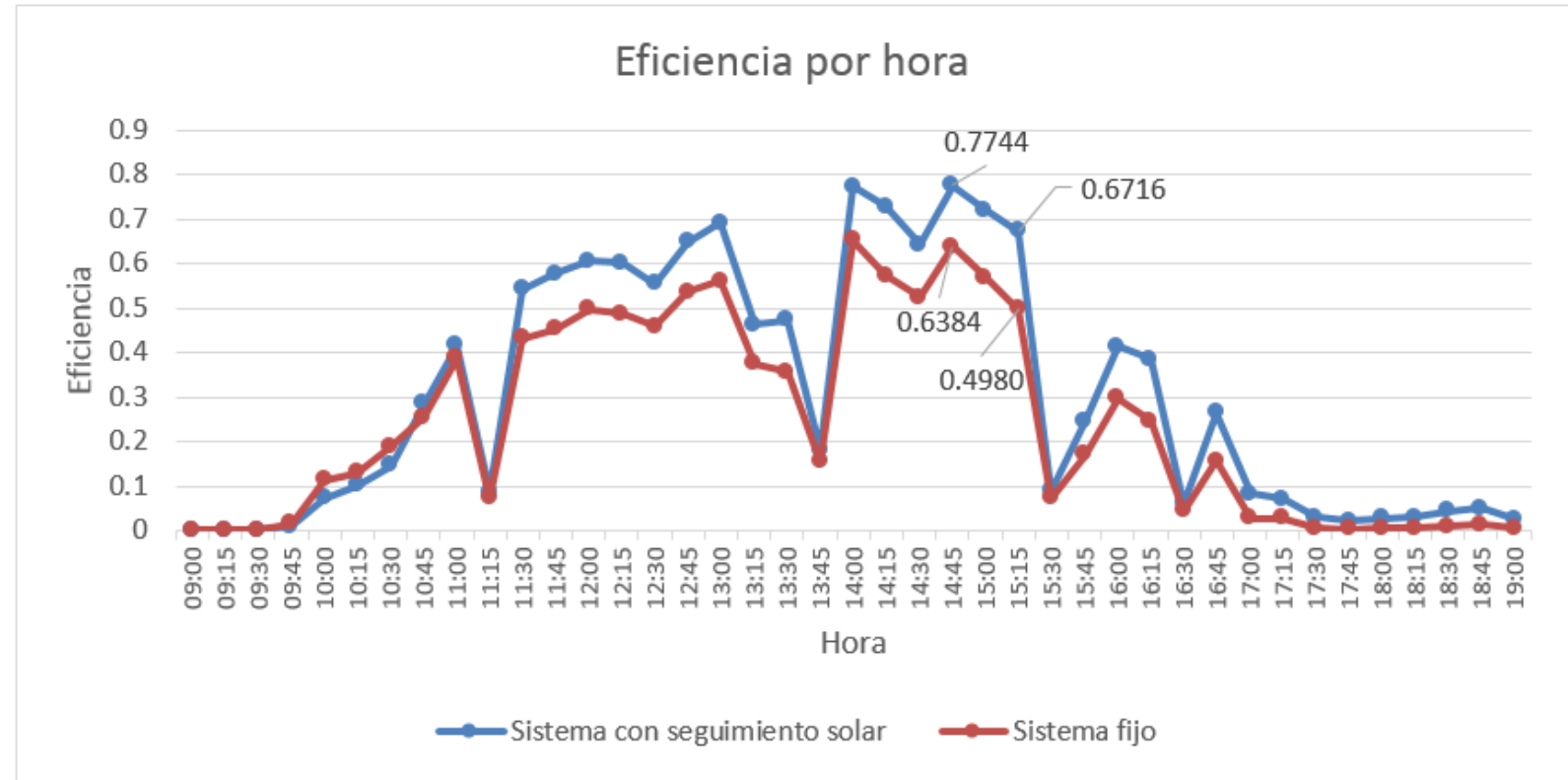

Fuente: Elaboración propia

Asimismo, el análisis de Tukey para las comparaciones de las medias de los dos sistemas demuestra una diferencia mínima significativa entre ambos, ver figuras 12 (a) y 12 (b).

Figura 12. Gráficos de la diferencia mínima significativa (Tukey) entre a) el seguidor y b) el sistema fijo

a) Seguidor Solar

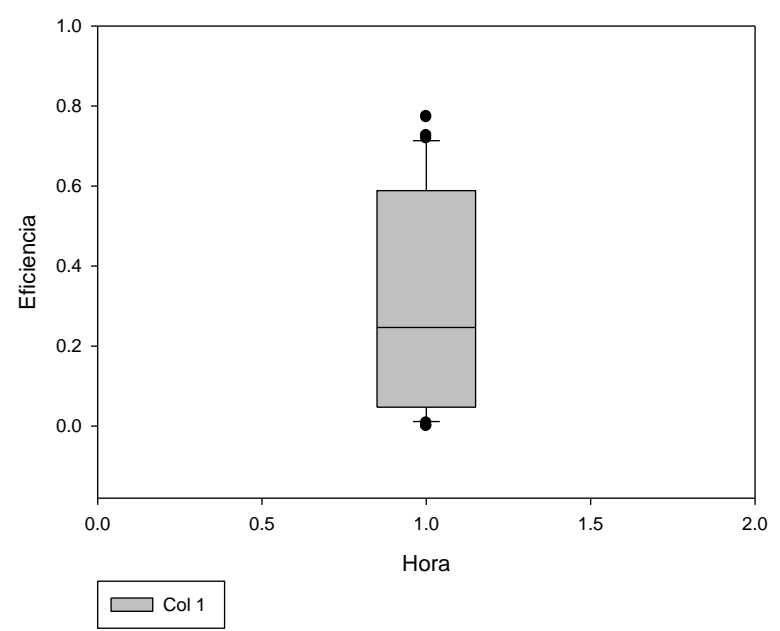

b) Sistema Fijo

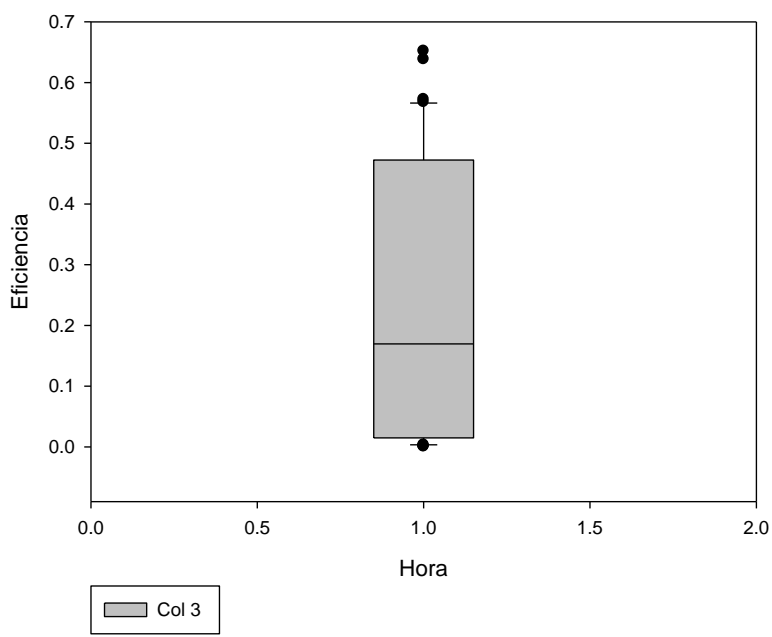

Fuente: Elaboración propia 


\section{Revista Iberoamericana de las Ciencias Biológicas y Agropecuarias}

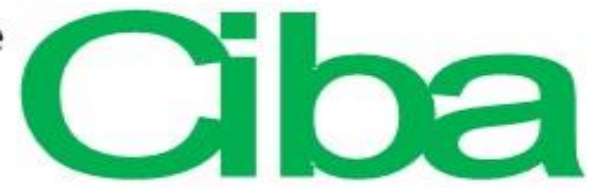

\section{Día soleado}

En esta investigación se consideró como día soleado el 14 de marzo de 2017 debido a la casi nula nubosidad en la ciudad de Autlán de Navarro. La eficiencia obtenida por ambos sistemas durante ese día se muestra en la figura 13. En esta se observa que desde las 09:00 horas hasta las 10:15 horas el sistema fotovoltaico fijo generó mayor potencia eléctrica que el sistema con seguimiento solar debido a la razón ya señalada en el día nublado.

Asimismo, se aprecia que a partir de las 10:30 horas el sistema con seguimiento solar generó mayor potencia eléctrica que el sistema fijo. Asimismo, se nota que a las 13:15 horas el sistema con seguimiento solar consiguió su máxima eficiencia (96.67 \%), mientras que a las 15:30 horas registró la mayor eficiencia en comparación con el sistema fijo (27.62 \%).

En cuanto a la eficiencia promedio generada ese día, el sistema con seguimiento solar registró $54.72 \%$, mientras que el sistema fijo consiguió $43.17 \%$ de promedio. Este dato demuestra que en días soleados el sistema con seguimiento solar es más eficiente que el sistema fijo, y que ambos sistemas son más eficientes esos días que durante los días nublados o parcialmente nublados.

El comportamiento de ambas gráficas (desde las 12:30 horas hasta las 18:15 horas) indica un aumento considerable en la eficiencia del sistema con seguimiento solar en comparación con el sistema fijo. Esto se debió a que durante el día no se presentaron nubosidades que propiciaran cifras similares de energía en los dos sistemas.

Cabe mencionar que durante el periodo del estudio el sistema fotovoltaico fijo no se encontraba alineado de acuerdo con la altitud solar correspondiente, pues en ningún intervalo cercano al medio día (incluso del día soleado) ambos sistemas fotovoltaicos lograron una eficiencia similar. Esto revela que en ningún momento de los días del experimento los rayos de sol estuvieron alineados de manera perpendicular al panel fotovoltaico fijo. 


\section{Revista Iberoamericana de las Ciencias Biológicas}

y Agropecuarias

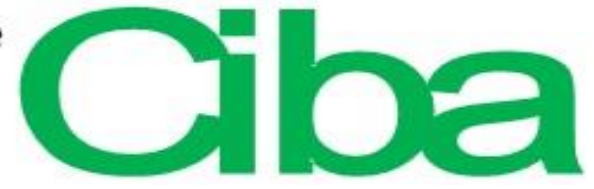

Figura 13. Gráfica del comportamiento de la eficiencia lograda el día 14 de marzo de 2017

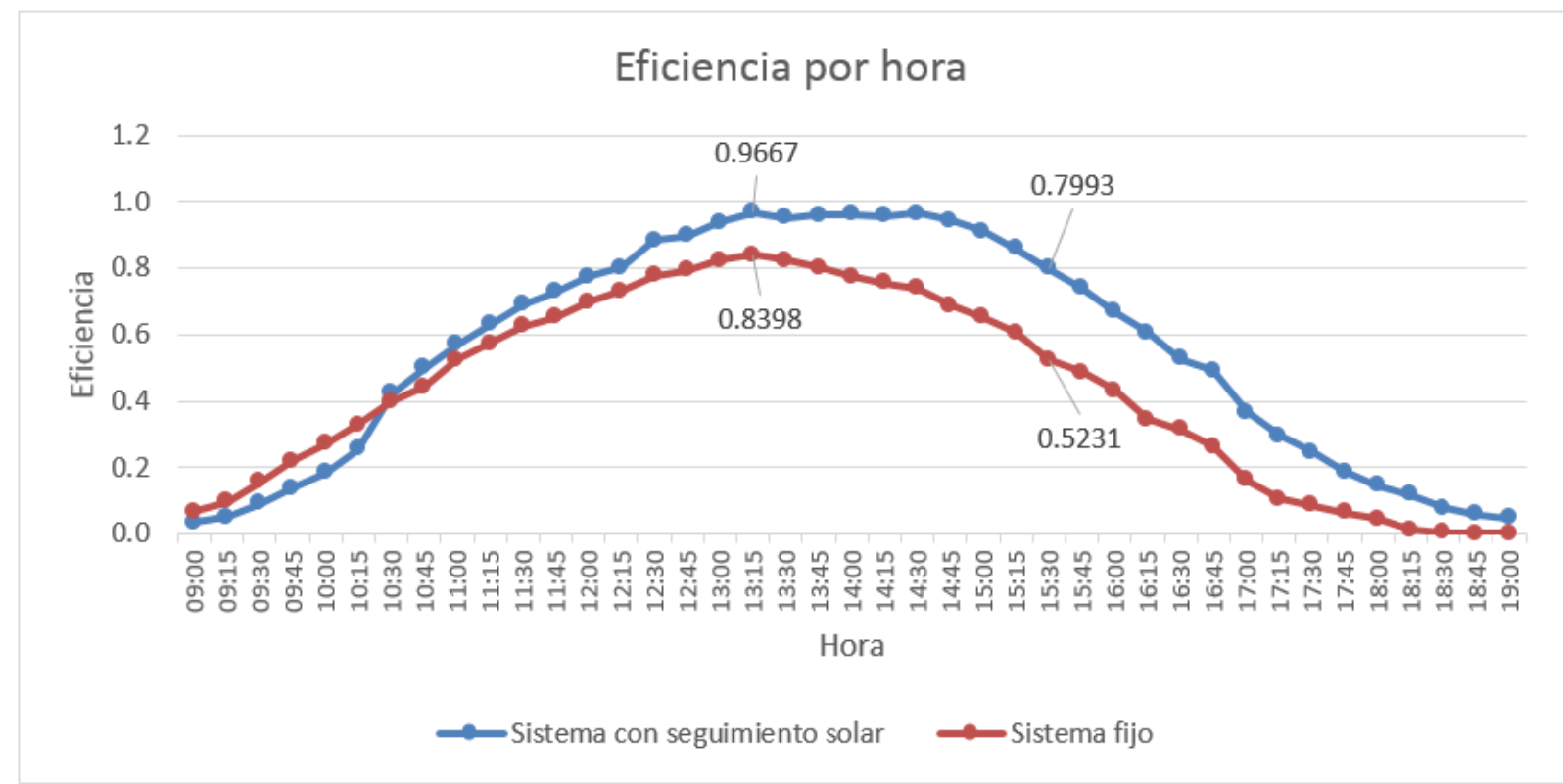

Fuente: Elaboración propia

Asimismo, el análisis de Tukey para las comparaciones de las medias de ambos sistemas arrojó una diferencia mínima significativa entre ellos, esto se observa en las figuras 14 (a) y 14 (b). Figura 14. Gráficos de la diferencia mínima significativa (Tukey) entre a) el seguidor solar y b) el sistema fijo

a) Seguidor Solar

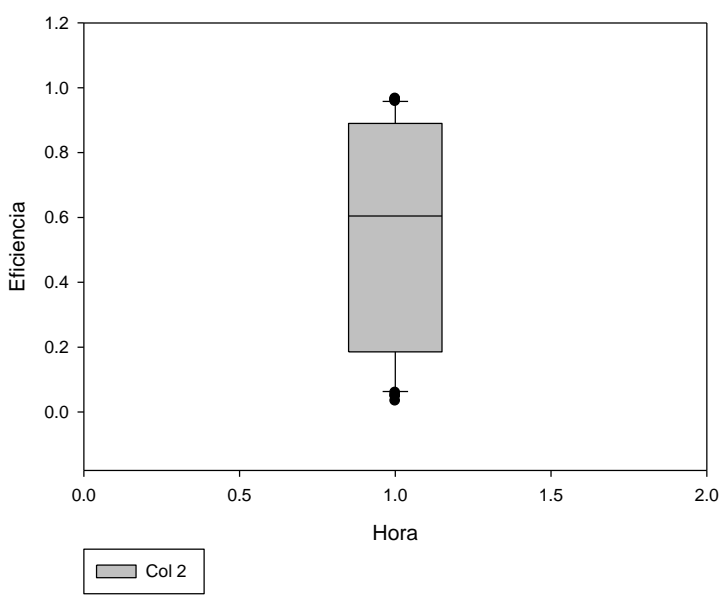

b) Sistema Fljo

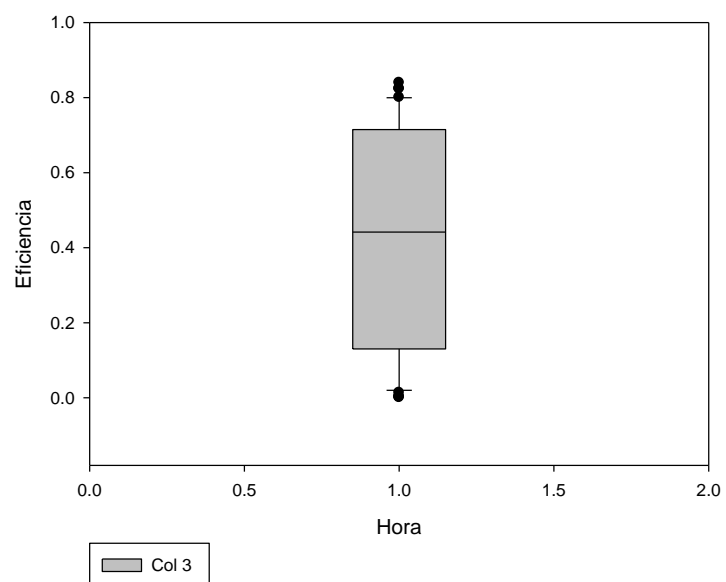

Fuente: Elaboración propia 


\section{Revista Iberoamericana de las Ciencias Biológicas y Agropecuarias}

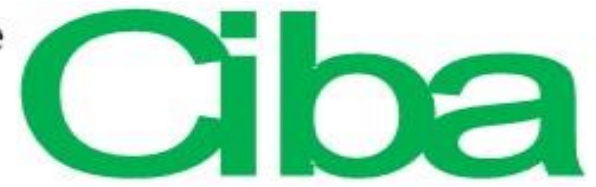

ISSN: $2007-9990$

\section{Periodo de 29 días}

En la figura 15 se aprecia el promedio de las eficiencias obtenidas por ambos sistemas durante el periodo experimental (del 15 de febrero al 15 de marzo de 2017). En la figura se observa que durante todos los días del estudio el sistema fotovoltaico con seguimiento solar generó más potencia eléctrica que el sistema fotovoltaico fijo. Asimismo, el 14 de marzo el sistema con seguimiento solar alcanzó una mayor eficiencia (54.72 \%) en comparación con los demás días, y ese mismo día consiguió la mayor eficiencia (11.55 \%) en comparación con el sistema fijo.

En cambio, el 27 de febrero el sistema con seguimiento solar registró la menor eficiencia $(13.63 \%)$. Asimismo, el 16 de febrero el sistema con seguimiento solar obtuvo la menor eficiencia (4.26\%) en comparación con el sistema fijo.

Por otra parte, la eficiencia promedio alcanzada por el sistema con seguimiento solar durante el periodo experimental fue de $33 \%$, mientras que el sistema fijo logró una eficiencia promedio de $26.28 \%$. Esto permite deducir que la eficiencia del sistema con seguimiento solar (durante el periodo experimental) fue mayor que la del sistema fijo en $7 \%$, aproximadamente.

Cabe destacar que las caídas en la eficiencia de ambos sistemas (figura 15) se halla en días nublados (p. ej., el 27 de febrero de 2017) o parcialmente nublados (p. ej., el 19 de febrero de 2017).

Figura 15. Gráfica de la eficiencia promedio diaria durante el periodo experimental

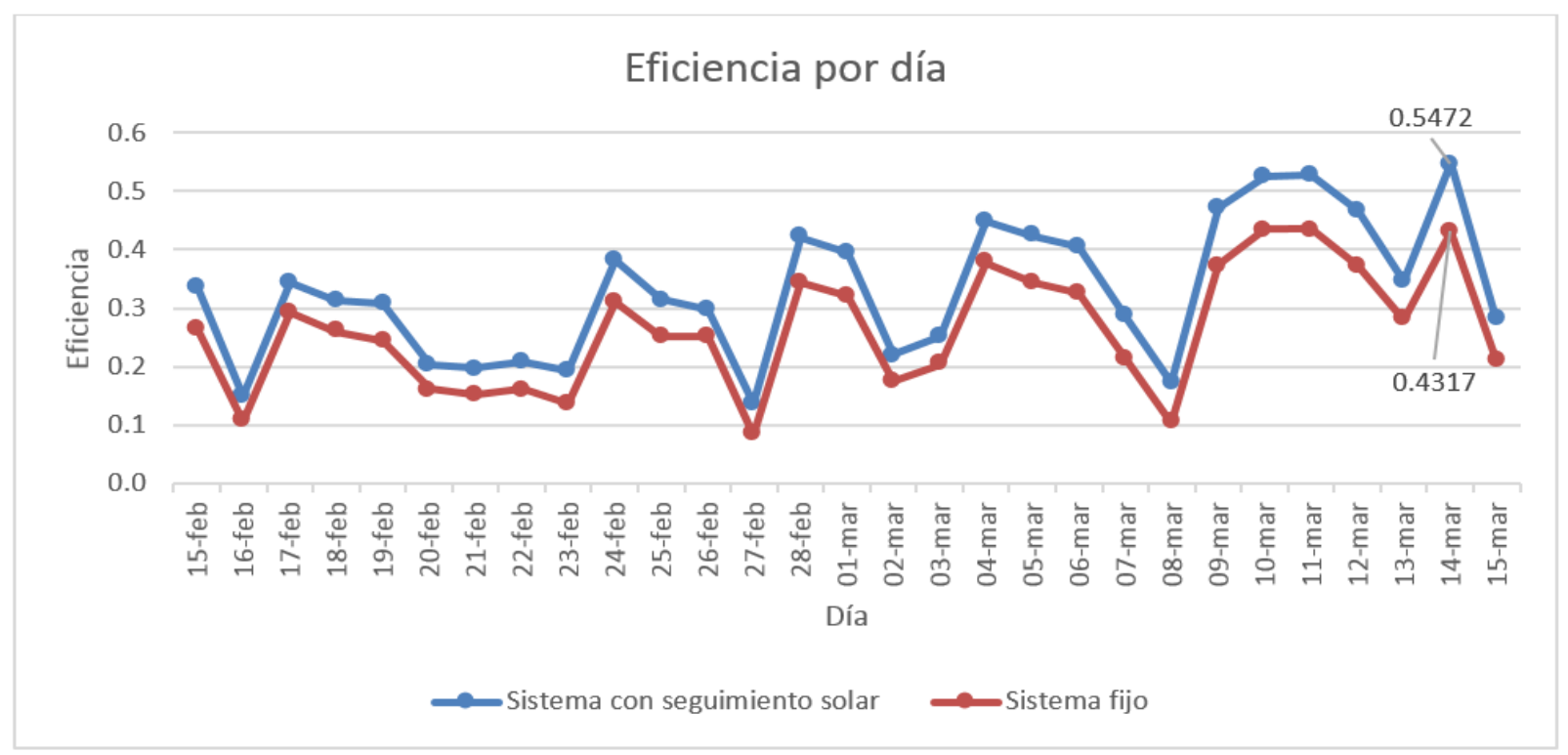

Fuente: Elaboración propia 


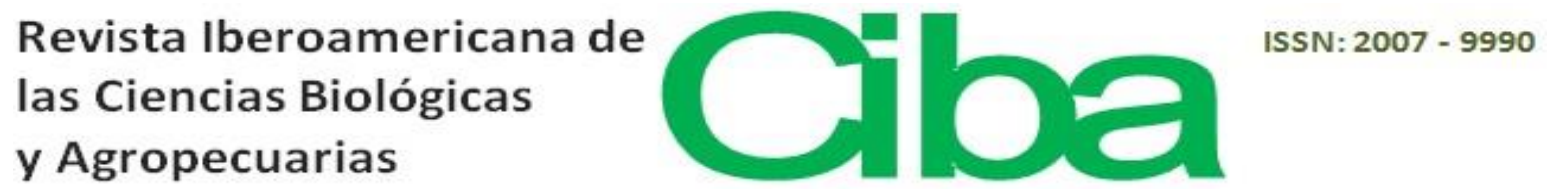

Asimismo, el análisis de Tukey para las comparaciones de las medias de ambos sistemas demostró una diferencia mínima significativa entre ellos, esta diferencia se observa en las figuras 16 (a) y $16($ b).

Figura 16. Gráficos de la diferencia mínima significativa (Tukey) entre a) el seguidor solar y b) el sistema fijo

a) Seguidor Solar

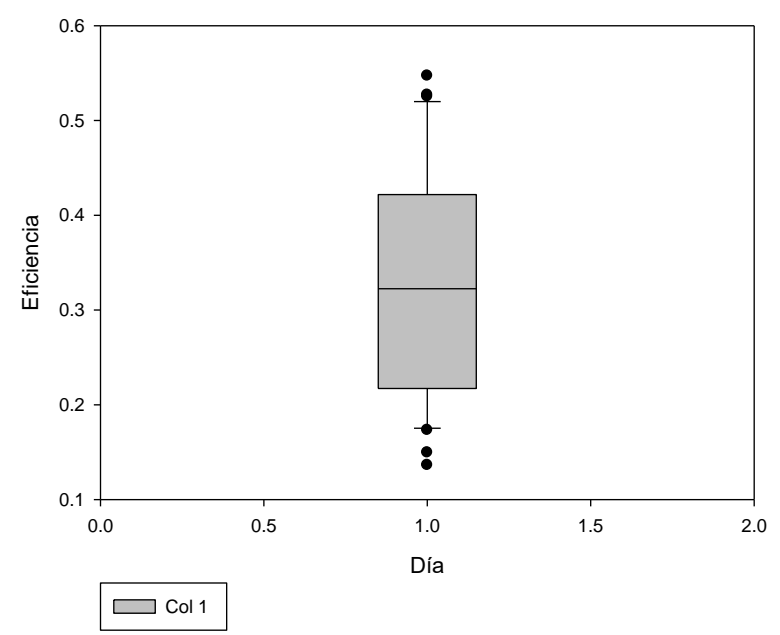

b) Sistema Fijo

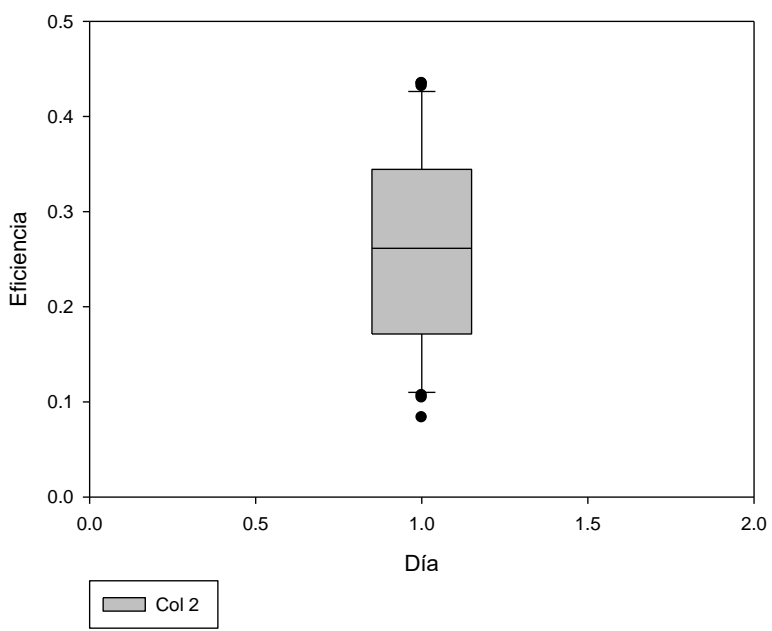

Fuente: Elaboración propia

Al realizar el análisis de comparativa de grupos (Anova) entre ambos sistemas, los datos obtenidos durante el periodo experimental de 29 días arrojaron los siguientes resultados, los cuales se muestran en la tabla1. 


\section{Revista Iberoamericana de las Ciencias Biológicas y Agropecuarias}

Tabla 1. Resultado del análisis de comparativa de grupos (Anova) entre ambos sistemas

\begin{tabular}{|c|c|c|c|c|c|c|}
\hline Nombre del grupo & $\mathbf{N}$ & Missing & Mean & Std Dev & SEM & \\
\hline Col 1 & 29 & 0 & 0.330 & 0.117 & 0.0213 & \\
\hline Col 2 & 29 & 0 & 0.263 & 0.101 & 0.0184 & \\
\hline \multicolumn{2}{|l|}{ Fuente de variación } & DF & SS & MS & $\mathbf{F}$ & $\mathbf{P}$ \\
\hline \multicolumn{2}{|l|}{ Entre grupos } & 1 & 0.0677 & 0.0677 & 5.700 & 0.020 \\
\hline \multicolumn{2}{|c|}{ Prueba de normalidad: } & \multicolumn{5}{|c|}{ aprobada $(\mathrm{P}=0.241)$} \\
\hline \multicolumn{2}{|c|}{ Prueba de varianza igual: } & \multicolumn{5}{|c|}{ aprobada $(P=0.418)$} \\
\hline
\end{tabular}

Fuente: Elaboración propia

Las diferencias de los valores medios entre los grupos de tratamiento son mayores de lo que cabría esperar por azar, pues hay una diferencia estadísticamente significativa $(\mathrm{P}=0.020)$. En otras palabras, existe mayor eficiencia en el sistema con seguimiento solar que en el sistema fijo, ya que hay una diferencia significativa entre ambos sistemas.

\section{Conclusiones}

El sistema fotovoltaico fijo generó una mayor cantidad de energía eléctrica en las primeras horas de cada día (antes de las 10:30 horas) en comparación con el sistema fotovoltaico de seguimiento solar. Esto debido a que con la puesta del sol, el panel fotovoltaico con seguimiento solar terminaba orientado hacia el poniente.

En los periodos donde se presentó una intensa nubosidad, la caída de eficiencia en ambos sistemas fotovoltaicos mostró aproximadamente el mismo valor. En otras palabras, en esos intervalos la eficiencia fue similar para los dos sistemas.

La máxima eficiencia alcanzada por ambos sistemas fotovoltaicos se obtuvo a las 13:15 horas, alcanzando valores de $96.67 \%$ de eficiencia en el sistema con seguidor solar y de $83.98 \%$ de eficiencia en el sistema fijo. Por otro lado, la mayor diferencia en la eficiencia obtenida entre ambos sistemas fotovoltaicos se obtuvo a las 15:30 horas.

Durante el periodo experimental de 29 días, los resultados obtenidos demostraron que, la eficiencia promedio alcanzada por el sistema con seguimiento solar fue de $33 \%$, mientras que con 


\section{Revista Iberoamericana de las Ciencias Biológicas y Agropecuarias}

el sistema fijo fue de $26.28 \%$, es decir, el sistema con seguimiento solar logró ser más eficiente que el sistema fotovoltaico fijo en un $7 \%$, aproximadamente.

\section{Referencias}

Ahmet Senpinar, M. C. (2012). Evaluation of power output for fixed and two-axis tracking PVarrays. Applied Energy, 92(1), 677-685.

Arroyo Romero, G. y Cortés Montes de Oca, L. A. (2015). Diseño y construcción de un mecanismo Automatizado para el seguimiento de la trayectoria solar (tesis de pregrado). Universidad Nacional Autónoma de México, Ciudad de México, México.

Dick Hedberg, S. K. (2010). The World Needs a New Energy Paradigm. AMBIO: A Journal of the Human Environment, (1), 1-10.

Domínguez González, H. (2012). Diseño de un sistema fotovoltaico para la generación de energía eléctrica en el COBAEV 35 Xalapa (tesis de maestría). Universidad Veracruzana, Veracruz, México.

Enríquez, J. M., Andújar, J. M. and Bohórquez, M. A. (2010). A reliable, fast and low cost maximun power point tracker for photovoltaic applications. Solar Energy, 84(1), 79-89.

Escobar Mejía, A., Holguín Londoño, M. y Osorio R., J. C. (2010). Diseño e implementación de un seguidor solar para la optimización de un sistema fotovoltaico. Scientia et Technica, (44), 245-250.

Grupta, A. (2011). Increasing Efficiency of the Photovoltaic System of Mobile Robotic Platforms for Military Application and Exploration. IEEE, (1), 1-8.

Iglesias, J. M. y Morales, J. (2013). Dimensionado de un sistema térmico solar mediante simulación y su validación energética. Ingeniería Energética, 34(1), 55-65.

International Energy Agency [IEA]. (2010). Key world energy statistics. Recuperado de http://es.scribd.com/doc/69552521/IEA-Key-World-Energy-Statistics

International Organization for Standardization [ISO]. (2011). ISO 50001 - Energy management. Recuperado de https://www.iso.org/iso-50001-energy-management.html

Kahn, N. A. (2012). Measuring The Light Intensity Of A Hybrid Powered CFL And LED Lighting Using 3D Electronic Vision In Rotation Of The Solar Panel. IEEE, (1), 111-115. 


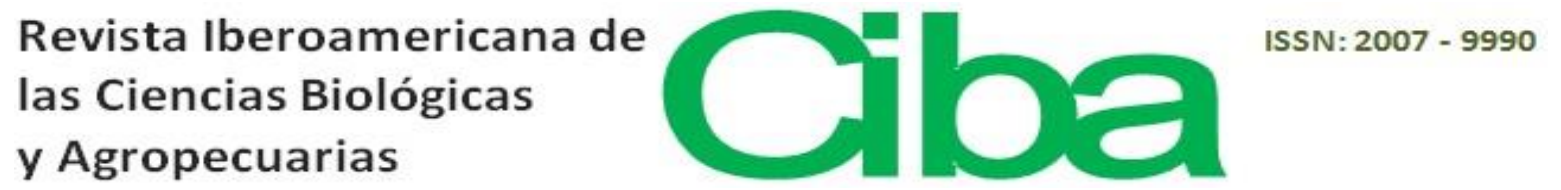

Koussa, A. C. (2011). Measured and modelled improvement in solar energy yield from flat plate photovoltaic systems utilizing different tracking systems and under a range of environmental conditions. Applied Energy, (88), 1756-1771.

Machado Toranzo, N., Lussón Cervantes, A., Leysdian Oro Carralero, L., Bonzon Henríquez, J. y Escalona Costa, O. (2015). Seguidor solar, optimizando el aprovechamiento de la energía solar. Ingeniería Energética, 36(2), 190-199.

Mousazadeh, H., Keyhani, A., Javadi, A., Mobli, H., Abrinia, K. and Sharifi, A. (2009). A review of principle and sun-tracking methods for maximizing solar systems output. Renewable and Sustainable Energy Reviews, (1), 1800-1818.

Neha, H., Gugri, S., Mishra S. and Dubey, G. (2013). Advancements in solar based LED street light. International Journal of Advanced Research in Electrical, Electronics and Instrumentation Engineering, 2(5), 1880-1884.

Noa-Diéguez, L. Y., Álvarez-Sánchez, V. y Pérez-Rodríguez, R. (2015). Diseño paramétrico asistido por computadora de un seguidor solar fotovoltaico a un eje polar. Ciencias Holguín, 21(2), 1-12.

Núñez Flores, A. (2012). Viabilidad de calentadores solares de agua con seguidor de trayectoria solar (tesis de maestría). Benemérita Universidad Autónoma de Puebla, Puebla, México.

Panait, M. A. and Tudorache, T. (2008). A simple neural networks solar tracker for optimizing conversion efficiency in off-grind solar generators. Santander: ICREPQ. 


\section{Revista Iberoamericana de las Ciencias Biológicas y Agropecuarias}

\begin{tabular}{|c|c|}
\hline Rol de Contribución & Autor(es) \\
\hline Conceptualización & $\begin{array}{l}\text { Director del proyecto: Jorge Arturo Pelayo López. Participantes: Alfredo Luna } \\
\text { Soto, Francisco Bernabe Ramos, Benjamín Guzmán Flores. }\end{array}$ \\
\hline Metodología & Jorge Arturo Pelayo López. \\
\hline Software & $\begin{array}{l}\text { Director del proyecto: Jorge Arturo Pelayo López. Alumno participante: Rodolfo } \\
\text { Rojano Cobián. }\end{array}$ \\
\hline Validación & $\begin{array}{l}\text { Director del proyecto: Jorge Arturo Pelayo López. Participante: Alfredo Luna } \\
\text { Soto, Francisco Bernabe Ramos. Alumno participante: Rodolfo Rojano Cobián. }\end{array}$ \\
\hline Análisis Formal & $\begin{array}{l}\text { Director del proyecto: Jorge Arturo Pelayo López. Participante: Francisco } \\
\text { Bernabe Ramos. }\end{array}$ \\
\hline Investigación & $\begin{array}{l}\text { Director del proyecto: Jorge Arturo Pelayo López. Participantes: Alfredo Luna } \\
\text { Soto, Francisco Bernabe Ramos, Benjamín Guzmán Flores. Alumno participante: } \\
\text { Rodolfo Rojano Cobián. }\end{array}$ \\
\hline Recursos & Universidad de Guadalajara. \\
\hline Curación de datos & $\begin{array}{l}\text { Director del proyecto: Jorge Arturo Pelayo López. Participantes: Alfredo Luna } \\
\text { Soto, Francisco Bernabe Ramos, Benjamín Guzmán Flores. }\end{array}$ \\
\hline $\begin{array}{l}\text { Escritura - Preparación del } \\
\text { borrador original }\end{array}$ & $\begin{array}{l}\text { Jorge Arturo Pelayo López, Alfredo Luna Soto, Francisco Bernabe Ramos, } \\
\text { Benjamín Guzmán Flores. }\end{array}$ \\
\hline Escritura - Revisión y edición & $\begin{array}{l}\text { Jorge Arturo Pelayo López, Alfredo Luna Soto, Francisco Bernabe Ramos, } \\
\text { Benjamín Guzmán Flores. }\end{array}$ \\
\hline Visualización & $\begin{array}{l}\text { Jorge Arturo Pelayo López, Alfredo Luna Soto, Francisco Bernabe Ramos, } \\
\text { Benjamín Guzmán Flores. }\end{array}$ \\
\hline Supervisión & $\begin{array}{l}\text { Director del proyecto: Jorge Arturo Pelayo López. Participantes: Alfredo Luna } \\
\text { Soto, Francisco Bernabe Ramos, Benjamín Guzmán Flores. }\end{array}$ \\
\hline Administración de Proyectos & Jorge Arturo Pelayo López. \\
\hline Adquisición de fondos & Universidad de Guadalajara. \\
\hline
\end{tabular}

